\title{
An Embedded Module as "VIRTUAL TONGUE" for Voiceless
}

\author{
${ }^{1}$ SANKAR KUMAR S, ${ }^{2}$ JENITHA J, ${ }^{3}$ NARMADHA I, ${ }^{4}$ SUGANYA A \\ ${ }^{1}$ Research Scholar, ${ }^{2,3,4}$ Final year UG \\ Department of Electronics and Communication Engineering \\ Velammal College of Engineering and Technology, Madurai-625009, Tamil Nadu
}

\begin{abstract}
There are several human disabilities in nature of which speech impaired people find difficulty in communicating with others, which is very important to convey their messages without speech. In this paper, to make them self reliable and independent, with the advent of embedded systems technology an embedded handheld icon based assistive device as "Virtual Tongue" for Voiceless, which speaks for severely speech disordered people by simply pressing icons appropriately to fulfill their needs, is proposed. This proposed module comprises a microcontroller based player to play voice messages, Secure Digital (SD) card reader, Universal Serial Bus (USB) port, icon based remote keypad, audio amplifier and speaker along with the benefits like portable, reliable, user friendly, affordable cost, low power consumption and of course speech in regional language with good clarity. The proposed system is designed to produce speech regardless of time length, audible to the neighbors, based on the request from the user by pressing the icons thereby this module deserves inarticulate people. An extended version with a feature of converting text into voice by adding a circuit, with which any text fed through a keyboard can be converted into speech, is also discussed.
\end{abstract}

\section{KEYWORDS}

Speech impaired, assistive device, voice synthesizer, text to speech

\section{INTRODUCTION}

In the recent years, there is a rapid increase in the number of speech - disabled victims due to several reasons like by birth, oral diseases, accidents, etc... and need for the Electronic Assistive Technology (EST) also increases particularly among physically impaired. Speech impairment is a communication problem in which the normal speech is disrupted due to articulation problems, because they are rendered helpless and unable to communicate with the outside world. To facilitate an easy communication for a verbally challenged people and overcome the hindrances in efficient communication, researchers have been developing ESTs and many of them are based on sign language, which is the language used by deaf and dump people for their communication. Sign language based ESTs are successful among the people those who are well versed with sign language and also less percentage of speech impaired community is familiar with sign language. Most of speech impaired people are not aware of this language and a considerable percentage of them are illiterate. In this paper, an embedded assistive aid, which acts as virtual tongue, developed for the people with communication complexity, to have a successful communication like one who without any disability through speech by handling the icons appropriately provided in the developed handheld module is presented. 
Idea behind the development of this module is at first to speak for the voiceless people in order to fulfill their basic needs thereby to make them self reliable and independent. The developed module produces voice which is audible to the neighbors of the user so that they can react (or) respond for the voice message. The advantages of this module are user friendly, affordable cost, portable, low power consumption and of course no skill requirement to operate because of its icon based keypad. Since the voice message is available from SD card, the most important benefit of this module is regardless of time any length and any language voice message can be stored and played. Because the assistive devices which speak for voiceless, developed so far are with limited time messages and are not having the feature of multilingual. But, in this module, according to the region of the user just by changing the SD card alone, the same module can be used to have successful voice communication.

Finally, in the enhanced version which can be utilized by literate but with speech disorder, instead of using collection of language specific, the user can convert text-to-speech through keyboard provided with this module, is also discussed.

\section{EXISTING METHODS}

An Electronic Speaking Glove is designed to facilitate an easy communication for a verbally challenged people, in which the gestures from the gloves are converted into a synthesized speech to convey an audible message to others. The glove is internally equipped with multiple flex sensors that are made up of bend-sensitive resistance elements.

Articulograph sensor AG-500 based system is placed on the lips of the user was developed. The sensor calculates the distance between the various reference points assigned to the mouth. The distance is mapped with equivalent SAMPA code which is fed as an input to the voice generating software and output is obtained in audio form.

A vocal cord vibration switch was developed which uses accelerometer sensors to detect the vibrations from the vocal cord. The vocal cord vibration due to speech are considered as high periodic signals and the vibrations produced due to coughs and swallows are considered as low periodic signals. These low periodic signals are filtered and the high periodic signals are allowed to activate the switch which can be connected to computers, or communication devices for further processing.

The digital speech aid, which is an electronic device developed for elimination or reduction of stuttering, which uses digital signal processing circuitry, to elicit the desired response characteristics of the feedback signal.

Kathamala, a Voice Output Communication Aid (VOCA) that uses ISD4004 ChipCorder which provides single-chip record/playback solutions for 8-16 minute messaging along with a Microcontroller to provide voice production at low cost.

An assistive aid for speech impaired that uses HM2007, a speech processor IC after trained by individual voice and developed with PIC Microcontroller to playback the stored words based on the vibration from acoustic plate.

The methods which exist related to this work are with several limitations (especially time limit) and involve complexity along with high cost. To overcome, it is decided to design and develop a handheld user friendly embedded module to speak with no time limit for them at an affordable 
cost without any complexity in handling the module to make them self reliable, happy and independent resulting Virtual Tongue.

\section{PROPOSED SYSTEM}

In this paper, EST is designed and implemented as virtual tongue which will help the people whom are unable to speak or having difficulties in speaking. A system-on-chip is used which contains a microcontroller along with MP3 decoder, control program, $\mathrm{I}^{2} \mathrm{C}$ controller for this application along with a IR remote keypad, SD card reader, USB port and an audio amplifier with speaker. Block diagram of the proposed system is shown in Figure.1.

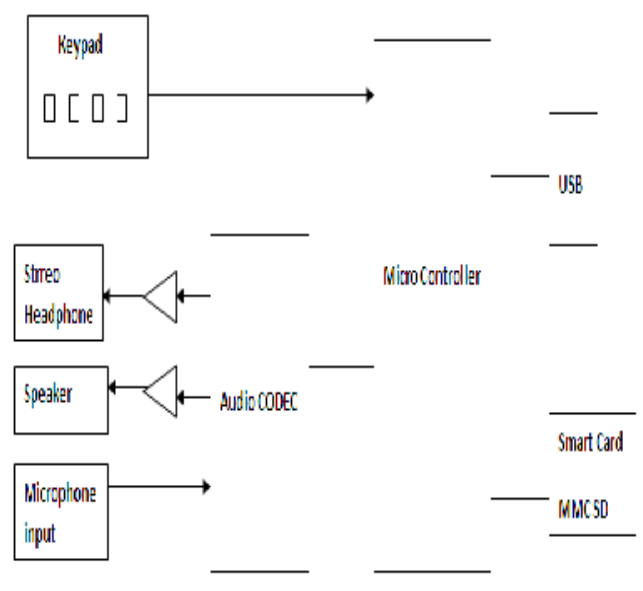

Figure.1 Block diagram of the proposed system

The system is equipped with icon based user friendly keypad. Icons will be selected by the user and based on the user's request the pre-programmed controller, will select appropriate voice track from the SD card (or) USB port and then the same will be played through audio amplifier to produce audio output.

\section{II.MPLEMENTATION}

The idea behind this proposed system is at first tried with APR9600, which is a 60 seconds recording and playback voice IC. Though it has multi-level analog storage, the total time length of stored voice should not exceed 60 seconds, thereby more divisions lead to less message length and the clarity of the audio output found not clear. Then, in order to increase the message length, studied about ISD4004 ChipCorder ${ }^{\circledR}$ which provides 3 volt single-chip record / playback solutions for 8-16 minute messaging. In both the cases, the difficulties inferred are the limited time length and requirement of skill to record / play a message.

To overcome the time length limit, i.e., to store voice message regardless of time and high quality audio output with clarity, it is decided to introduce the MP3 principle in our system. 


\section{II.SD MEMORY CARD}

The SD card system is a new mass-storage system based on innovations in semiconductor technology. This SD card is highly integrated flash memory with serial and random access capability. It is accessible via a dedicated serial interface optimized for fast and reliable data transmission. This interface allows several cards to be stacked by through connecting their peripheral contacts. SD card allows the design of inexpensive players and drivers without moving parts. The block diagram of SD memory card is shown in Figure.2.

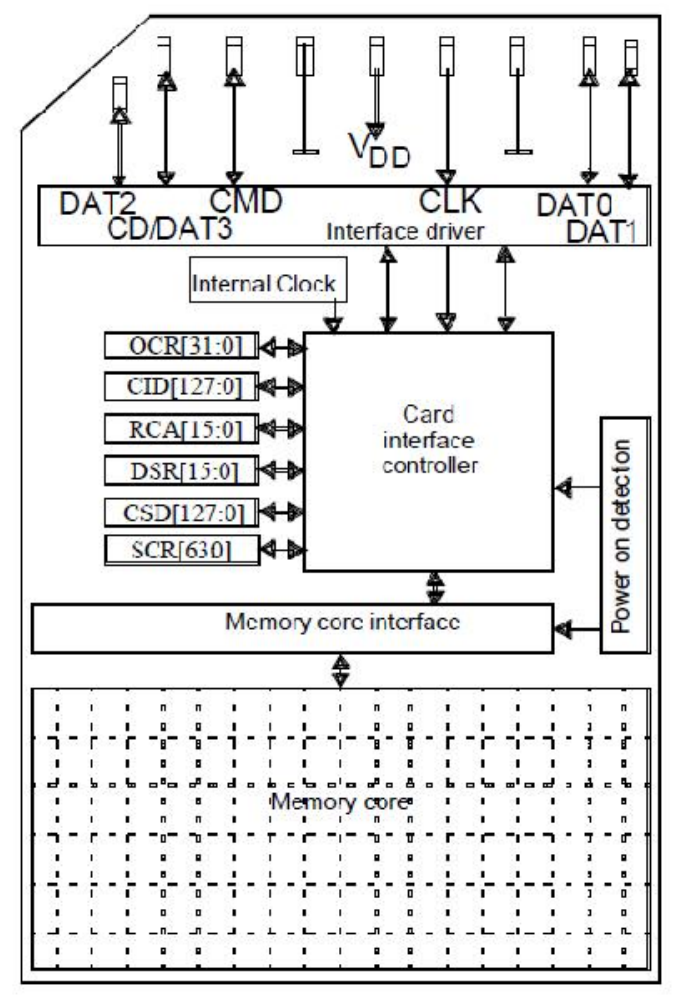

Figure.2 Block diagram of SD Memory card

All units these SD card are clocked by an internal clock generator. The interface driver unit synchronizes the DAT and CMD signals from external CLK to the internal used clock signal. The card is controlled by the six line SD card interface containing the signals: CMD, CLK, DAT0 DAT3. After power up by default, the SD card will use only DAT0.

These SD Card interface can operate in two different modes:

. SD Card mode

. SPI mode

Host system can choose either one of modes. SD Card mode allows the 4-bit high performance data transfer. SPI mode allows easy and common interface for SPI channel. 


\section{II.USB}

USB is a simple and flexible interface between a system and peripherals. USB allows hot swapping. The "hot-swapping" means that the devices can be plugged and unplugged without rebooting the computer or turning off the device. That means, when plugged in, everything configures automatically and when unplugged, the host will detect its absence and automatically unload the driver. USB is a serial protocol and physical link with two pair of wires, with which transmits all data differentially on a single pair of wires. Another pair provides power to downstream peripherals. The block diagram of USB Device controller and USB connection diagram are shown in Figure.3 (a) \& (b) respectively.

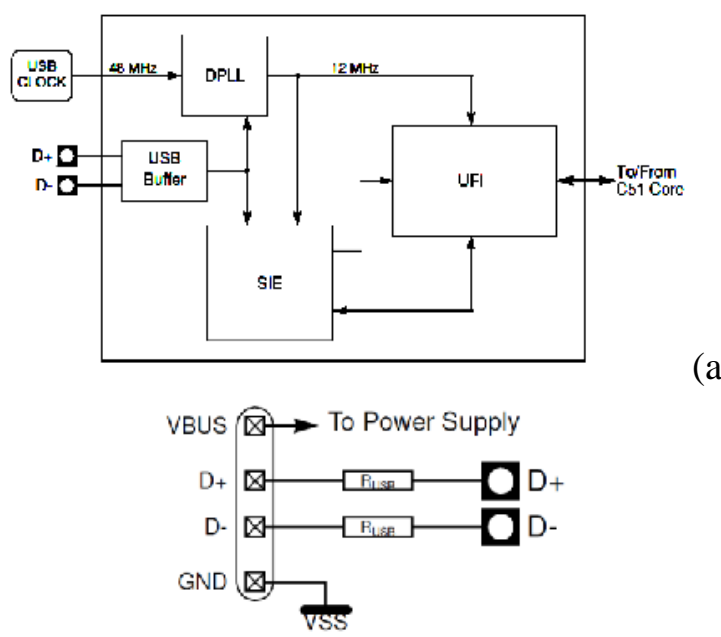

(a)

(b)

Figure.3 (a) Block diagram of USB Device Controller (b) USB Connection

The USB device controller provides the hardware to interface a USB link to a data flow stored in a double port memory. It requires a $48 \mathrm{MHz}$ reference clock provided by the clock controller. This clock is used to generate a $12 \mathrm{MHz}$ Full Speed bit clock from the received USB differential data flow and to transmit data according to full speed USB device tolerance. Clock recovery is done by a Digital Phase Locked Loop (DPLL) block.

The Serial Interface Engine (SIE) block performs NRZI encoding and decoding, bit stuffing, CRC generation and checking, and the serial-parallel data conversion. The Universal Function Interface (UFI) controls the interface between the data flow and the Dual Port RAM, but also the interface with the C51 core itself.

When a USB peripheral device is first attached to the network, a process called enumeration process gets started. This is the way by which the host communicates with the device to learn its identity and to discover which device driver is required. The enumeration starts by sending a reset signal to the newly connected USB device. In other words, when the host is powered up and a device connected or removed from the network, the enumeration process is initiated. 


\section{II.VTV CONTROLLER}

The MP3 voice messages stored in removable SD card (or) USB drive are to be played based on the request from the user appropriately. To accomplish the process, a programmable device is required to process the user's request, which is named as VTV controller, shown in Figure.4. This controller contains typical 80C31 microcontroller core with a dedicated MP3 decoder with full audio interface. Built-in 20-bit stereo audio DAC, $440 \mathrm{~mW}$ mono Audio power amplifier, IR receiver, Full speed USB controller, PLL for Audio clock and USB clock, I ${ }^{2} \mathrm{C}$ (Inter Integrated Circuit) bus controller and standard full duplex UART.

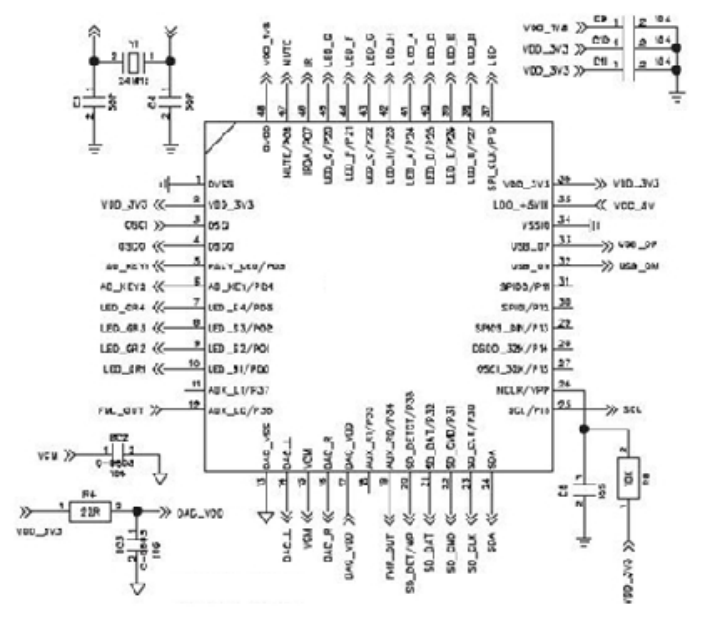

Figure.4 VTV Controller

$\mathrm{I}^{2} \mathrm{C}$ bus provides good support for communication with various slow, on-board peripheral devices that are accessed intermittently. Most available $\mathrm{I}^{2} \mathrm{C}$ devices operate at speeds up to $400 \mathrm{Kbps} . \mathrm{I}^{2} \mathrm{C}$ is appropriate for interfacing to devices on a single board, and can be stretched across multiplboards inside a closed system. $\mathrm{I}^{2} \mathrm{C}$ is a two-wire serial bus.

\section{II.AUDIO AMPLIFIER}

The proposed module is developed to produce voice for the voiceless. But, the audio output from the VTV controller is $440 \mathrm{~mW}$, which not audible to the neighbor. So, an additional audio amplifier circuit is introduced with D6283 Audio IC, a 4.6W dual audio power amplifier, whose output is audible to a neighbor of this module's user. D6283 is an audio power IC with built-in two channels developed for portable audio players. Also, it has built-in thermal shut down protection circuit and low popping noise at power ON. The circuit diagram of $4.6 \mathrm{~W}$ audio power amplifier is shown in Figure.5. 


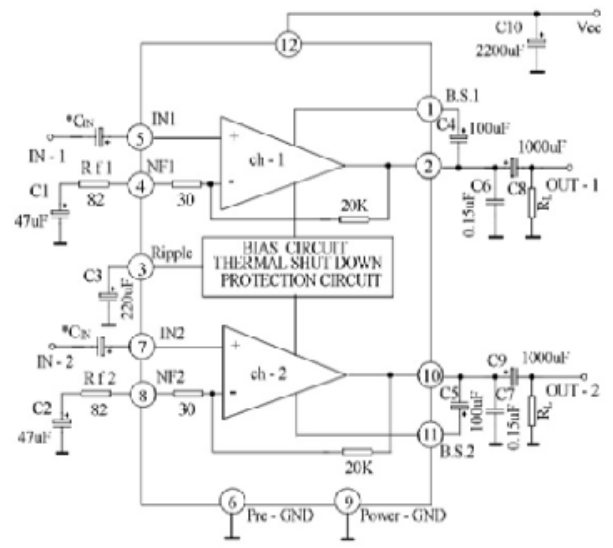

Figure.5. 4.6 W Audio Power Amplifier

\section{II.IR REMOTE CONTROL}

In this module, an icon based keypad, shown in Figure.6 is used as input device to select the request in order to produce appropriate voice message. Since the VTV controller has built-in IR receiver, a wireless transmitter is used with input keypad, which is an IR remote control on which the icons are provided.
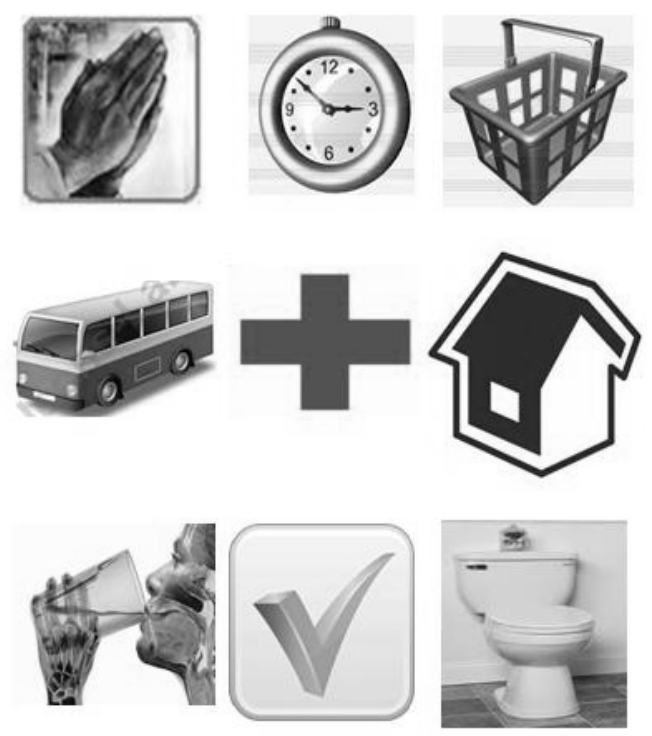

Figure.6. Icon based keypad

The remote controller itself called as transmitter. Infrared remote controller use invisible light pulses below the visible wavelength spectrum. There is a line of sight between the transmitter (light source) and the receiver (light detector).The operating range of IR remote control from 30 feet to 100 feet and allows the signal to penetrate on walls and glass cabinet enclosures. IR transmitter and receiver systems are inexpensive and are generally reliable.The carrier frequency 
of such infrared signals is typically in the order of around $38 \mathrm{kHz}$. Pushing a button on a remote control sets in motion a series of events that causes the controlled device to carry out a command.

\section{II.RESULTS \& DISCUSSIONS}

The developed system Virtual Tongue for Voiceless is shown in Figure.7.

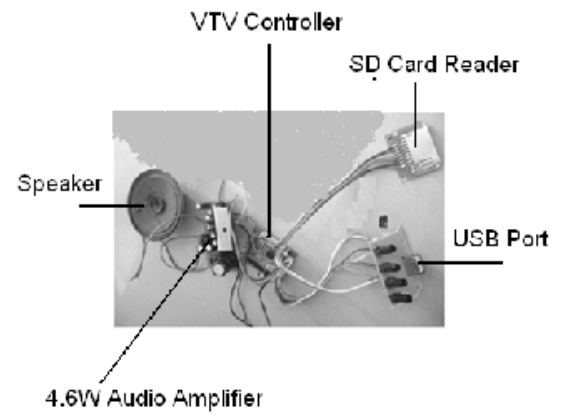

Figure.7. Virtual Tongue for Voiceless

Since, the audio files are MP3 format, the clarity of the voice is clear and of course regardless of the message length time.

To store the voice messages in MP3 format, with the help of online open source TEXT-TOSPEECH converter, the messages are converted into speech and are stored in SD card /USB drive. The advantage of this module is the voice messages can be stored in any language with the help of the above said converter and hence, this becomes a multi-lingual module for voiceless. In other words, having SD cards with messages in various languages, this module becomes multilingual just by changing the SD card alone.

\section{II.FUTURE WORK}

The developed handheld user friendly module is for people with speech disorder, for which no skill is required to operate and the limitation in this module is, the pre-recorded messages assigned for the icons on the keypad only played. But, this can be enhanced for skilled \& literate people by adding a text-to-speech IC TTS256 and any text fed through keyboard (may be a string, numbers etc.) can also be played.

\section{II.CONCLUSION}

The proposed system Virtual Tongue for Voiceless is developed with SD card reader, USB port, 4.6W Audio output and a remote control with icon based keypad. User friendly, portable, affordable cost and low power consumption are the striking features of this module.

\section{REFERENCES}

[1] "The amazing digital gloves that gives Voice to the Voiceless" by Praveen kumar S Havalagi ,Shruthi Urf Nivedita, International Journal of Advances in Engineering \& Technology, 2013

[2] "A Review of Literature on Hand Gesture Recognition for Indian Sign Language" by Neha V. Tavari,A. V. Deorankar, Dr. P. N. Chatur ,International Journal of Advance Research in Computer Science and Management Studies, 2013 
International Journal of Information Sciences and Techniques (IJIST) Vol.4, No.3, May 2014

[3] "Sign Language Recognition System For Deaf And Dumb People" by Sakshi Goyal, Ishita Sharma, Shanu Sharma,Amity University,Noida,Uttar Pradesh,India,2013

[4] "Development of an Assistive Aid for Speech Impaired "by P.Aparna, P.Mohanapriya, International Journal of Scientific and Research Publications, Chennai 2012.

[5] "SymbolPath: A Continuous Motion Overlay Module for Icon-Based Assistive Communication ",by Karl Wiegand,Rupal Patel,Northeastern University,USA,2012

[6] "Voice for the voiceless" by E.Jeevitha, M.R.Meenakshi, V.K.G.Kalaiselvi, Chennai, Computer Science \& Education (ICCSC), Augest 2011

[7] "Electronic Speaking Glove for speechless patients, a tongue to a dumb" by B.Ali,S.Munawwar, B.Nadeem,Sustainable Utilization and Development in Engineering and Technology, 2011

[8] "Dynamic Acoustic for Dumb Using Embedded Interface (DADEI)" by Srikkanth. G,Computer Modelling and Simulation, 2010

[9] "Translating the sign of dumb person using ARM processor" by Brindhu Kumari.A, Communication Control and Computing Technologies(ICCCCT),2010 